\title{
SOME SUFFICIENT CONDITIONS FOR UNIVALENCE
}

\author{
SUKHJIT SINGH \\ Department of Mathematics \\ Punjabi University \\ Patiala-147002 (Punjab) \\ Indi a \\ (Received May 5, 1989 and In revised form January 1, 1990)
}

\begin{abstract}
A new subclass $R(\alpha), 0<\alpha<1$, of the class $s_{t}(1 / 2)-t$ the class of starlike functions of order $1 / 2$ - is introduced and it is shown that $R(\alpha)$ is closed with respect to the Hadamard product of analytic functions. Some sufficient conditions for the normalized regular functions to be univalent in the unit disk $E$ are given.
\end{abstract}

KEY WORDS AND PHRASES. Convex function, close-to-convex function, starlike function of order $1 / 2$, univalent function, Hadamard product.

1980 AMS SUBJECT CLASSIFICATION CODE. $30 \mathrm{C} 45$.

1. INTRODUCTION.

Let $A$ denote the class of functions $f(z)=z+a_{2} z^{2}+\ldots$ which are regular in the unit disk $E=\{z /|z|<1\}$. We denote by $S$ the subclass of $A$ consisting of functions $f$ which are univalent in $E, K$ will stand for the usual subclass of $S$ whose members are convex in $E$. A function $f \varepsilon A$ is said to be close-to-convex in $E$ if and only if $\operatorname{Re}\left(f^{\prime}(z) / g^{\prime}(z)\right)>0, z \varepsilon E$, for some $g \in K$. Since $g(z) \equiv z$ is convex in E, the functions $f \varepsilon A$ which satisfy $\operatorname{Re} f^{\prime}(z)>0, z \varepsilon E$ are close-to-convex in $E$. It is well known that every close-to-convex function in $E$ is univalent in $E$. For a given $\alpha, 0<\alpha<1$, denote by $s_{t}(\alpha)$ the subclass of $s$ consisting of functions $f$ which satisfy the condition

$$
\operatorname{Re}\left(\frac{z f^{\prime}(z)}{f(z)}\right)>\alpha, z \varepsilon E
$$

$s_{t}(\alpha)$ is called the class of starlike functions of order $\alpha$. It is also well known that for $0<\alpha<\beta<1, S_{t}(\beta) \subseteq S_{t}(\alpha)$.

In the present paper we introduce a new subclass $R(\alpha)$ of the class $S_{t}(1 / 2)$ and prove that $R(\alpha)$ is closed with respect to convolution/Hadamard product of analytic functions. Some sufficient conditions are given for a function $f \varepsilon A$ to be in the class $\mathrm{S}$. 


\section{PRELIMINARIES.}

We shall need the following definitions and results. If $f(z)=\sum_{n=0}^{\infty} a_{n} z^{n}$ and $g(z)=\sum_{n=0}^{\infty} b_{n} z^{n}$ are regular in $E$, then their convolution/Hadamard product is the function ${ }^{n}$ denoted by $f * g$ and defined by the power series

$$
(f * g)(z)=\sum_{n=0}^{\infty} a_{n} b_{n} z^{n}
$$

Let $a, b$ and $c$ be any complex numbers with $c$ nefther zero nor a negative integer. Then the hypergeometric function $F(a, b ; c ;<)$ is defined in Rainville [1, p. 45] by

$$
F(a, b ; c ; z)=1+\sum_{n=1}^{\infty} \frac{(a)_{n}(b)_{n}}{(c)_{n} n !} z^{n}
$$

where $(\mu)_{n}$ is the Pochhammer symbol defined by

$$
(\mu)_{n}=\left\{\begin{array}{l}
1, \text { if } n=0 \\
\mu(\mu+1) \ldots(\mu+n-1), \text { if } n \in N=\{1,2,3, \ldots\} .
\end{array}\right.
$$

It is known that the series on the right in (2.2) is convergent for $z \varepsilon E$.

Now we define the function $\varphi(a, c)$ by

$$
(a, c ; z)=\sum_{n=0}^{\infty} \frac{(a)_{n}}{(c)_{n}} z^{n+1},(c \neq 0,-1,-2, \ldots ; z \varepsilon E) .
$$

From (2.2) and (2.4) we immediately get

$$
(a, c ; z)=2 F(1, a ; c ; z)
$$

LEMMA 2.1. [1, p. 47]. If $|z|<1$ and if $\operatorname{Re}(c)>\operatorname{Re}(b)>0$,

$$
F(a, b ; c ; z)=\frac{\Gamma(c)}{\Gamma(b) \Gamma(c-b)} \int_{0}^{1} t^{b-1}(1-t)^{c-b-1}(1-t z)^{-a} d t
$$

LEMMA 2.2. For a given real number $\alpha$, let

$f_{\alpha}(z)=\sum_{n=1}^{\infty} n^{-\alpha_{z} n}, z \in E$. Then $f_{\alpha}$ is convex whenever $\alpha>0$.

LEMMA 2.3. Let $f \in S_{t}(1 / 2)$ and $g \in S_{t}(\beta)$, where $1 / 2<\beta<1$. Then $f \star g$ is a member of $S_{t}(B)$.

LEMMA 2.2 is due to Lewis [2] and Lemma 2.3 follows the Corollary 1 in Lewis [3] by taking $\alpha=1 / 2$.

LEMMA 2.4. If $f \in K$, then $\operatorname{Re}(f(z) / z)>1 / 2, z \varepsilon E$. 
LEMMA 2.5. If $p(z)$ is analytic in $E, p(0)=1$ and $\operatorname{Re} p(z)>1 / 2, z \varepsilon E$, then for any function $F$, analytic in $E$, the function $P * F$ takes values in the convex hull of $F(E)$. Lemma 2.4. is due to Strohhäcker [4] and the assertion of Lemma 2.5 readily follows by using Herglotz' representation for $P(z)$.

3. THEOREMS AND THEIR PROOFS.

For $0<\alpha<1$, let $R(\alpha)$ denote the class of functions $f \varepsilon$ A which satisfy the condition

$$
\sum_{n=1}^{\infty} n^{\alpha} z^{n} * f(z) \varepsilon S_{t}\left(\frac{1+\alpha}{2}\right), z \varepsilon E .
$$

Clearly $R(0)=S_{t}(1 / 2)$ and $f \varepsilon R(1)$ if and only if $f(z) \equiv z$.

THEOREM 3.1. (i) If $0<\alpha<\beta<1$, then $R(\beta) \subseteq R(\alpha)$. (ii) $R(\gamma)$ is a subclass of $s_{t}(1 / 2)$ for every $\gamma>0$. $_{\infty}$

PROOF. Let $f(z)=z+\sum_{n=2}^{\infty} a_{n} z^{n} \varepsilon R(B)$ so that

$$
g(z)=\sum_{n=1}^{\infty} n^{\beta} z^{n} * f(z) \varepsilon s_{t}((1+\beta) / 2) \text {. }
$$

Now

$$
\begin{aligned}
\sum_{n=1}^{\infty} n^{\alpha} z^{n} * f(z) & =\left(\sum_{n=1}^{\infty} n^{\beta} z^{n} * f(z)\right) * \sum_{n=1}^{\infty} n^{\alpha-\beta} z^{n} \\
& =g(z) * k(z),
\end{aligned}
$$

where $k(z)=\sum_{n=1}^{\infty} n^{-(\beta-\alpha)} z^{n}$.

Since $\beta-\alpha \geqslant 0$, therefore by Lemma $2.2, k(z) \varepsilon K \subseteq S_{t}(1 / 2)$. In view of Lemma 2.3 , we now get from (3.2) and (3.3) that

$$
\left.g * k \in S_{t}((1+\beta) / 2) \subseteq S_{t}((1+\alpha) / 2), \quad \text { (as } \alpha<\beta\right)
$$

Hence from (3.3) and (3.1) we conclude that $f \in R(\alpha)$. This completes the proof of part (i). The proof of part (ii) follows immediately from part (i) and from the observation that $R(0)=s_{t}(1 / 2)$.

THEOREM 3.2. If $f$ and $g$ both belong to $R(\alpha)$, then $f * g$ also belongs to $R(\alpha)$. PROOF. Since $f \in R(\alpha)$, therefore

$$
h(z)=\sum_{n=1}^{\infty} n^{\alpha} z^{n} * f(z) \quad \varepsilon \quad s_{t}((1+\alpha) / 2)
$$

Now

$$
\begin{aligned}
\sum_{n=1}^{\infty} n^{\alpha} z^{n} *(f * g)(z) & =\left(\sum_{n=1}^{\infty} n^{\alpha} z^{n} * f(z)\right) * g(z) \\
& =h(z) * g(z) .
\end{aligned}
$$


Since $g \in R(\alpha) \subseteq S_{t}(1 / 2)$, therefore in view of Lemma 2.3 , we get, from (3.4) and (3.5) that

$$
h * g \in S_{t}((1+\alpha) / 2)
$$

which in turn implies that

$$
f \star g \in R(\alpha) \text {. }
$$

This completes the proof of our theorem.

THEOREM 3.3. If $f(z)=z+\sum_{n=2}^{\infty} a_{n} z^{n} \varepsilon A$ and satisfies the condition

$$
\operatorname{Re}\left[1+\sum_{n=2}^{\infty} n^{\alpha} a_{n} z^{n-1}\right]>0, \alpha>1, z \varepsilon E,
$$

then $\operatorname{Re} f^{\prime}(z)>0, z \in E$. Hence $f(z)$ is close-to-convex in $E$ and therefore univalent in E.

PROOF. We can write

$$
f^{\prime}(z)=1+\sum_{n=2}^{\infty} n a_{n} z^{n-1}=\left(1+\sum_{n=2}^{\infty} n^{\alpha} a_{n} z^{n-1}\right) *\left(1+\sum_{n=2}^{\infty} \frac{z^{n-1}}{n^{\alpha-1}}\right) \cdots
$$
Now by Lemma 2.2 , the function $k_{\alpha}(z)=z+\sum_{n=2}^{\infty}\left(z^{n} / n^{\alpha-1}\right)$ is convex for $\alpha>1$.
Therefore, in view of Lemma 2.4 ,

$$
\operatorname{Re} \frac{k_{\alpha}(z)}{z}=\operatorname{Re}\left[1+\sum_{n=2}^{\infty} \frac{z^{n-1}}{n^{\alpha-1}}\right]>1 / 2 .
$$

Thus, from (3.6), (3.7), (3.8) and Lemma 2.5, we conclude that Re $f^{\prime}(z)>0$.

THEOREM 3.4. Let $f \in A$ and let for $0<\beta<\alpha$, the condition

$$
\operatorname{Re}\left[(\varphi(\alpha, \beta ; z) * f(z))^{\prime}\right]>1 / 2, \quad z \varepsilon E,
$$

be satisfied. Then $\operatorname{Re} f^{\prime}(z)>0, z \varepsilon E$. Hence $f(z)$ is close-to-convex in $E$ and therefore univalent in $E$.

PROOF. The case when $\alpha=\beta$ is obvious, therefore we let $\beta<\alpha$. We can write

$$
\begin{aligned}
f^{\prime}(z) & =\left[\frac{\varphi(\alpha, \beta ; z)}{z} \star f^{\prime}(z)\right] *\left[\frac{\varphi(\beta, \alpha ; z)}{z}\right] \\
& =(\varphi(\alpha, \beta ; z) \star f(z))^{\prime} \star\left[\frac{\varphi(\beta, \alpha ; z)}{z}\right] .
\end{aligned}
$$

Now from (2.5) and Lemma 2.1, we have

$$
\frac{\varphi(\beta, \alpha ; z)}{z}=F(1, \beta ; \alpha ; z)=\frac{\Gamma(\alpha)}{\Gamma(\beta) \Gamma(\alpha-\beta)} \int_{0}^{1} t^{(\beta-1)}(1-t)^{\alpha-\beta-1}(1-t z)^{-1} d t
$$


Since $\operatorname{Re}\left[t^{\beta-1}(1-t)^{\alpha-\beta-1}(1-t z)^{-1}\right]>0$ for all $t, 0<t<1$ and for all $z$, $z \varepsilon E$, it follows that

$$
\operatorname{Re}\left[\frac{\varphi(\underline{\beta}, \alpha ; z)}{z}\right]>0, z \varepsilon E
$$

Form (3.9), (3.10), (3.11) and Lema 2.5 the assertion of the theorem now follows.

ACKNOWLEDGEMENTS. The author wishes to thank the referee for his valuable suggestions and comments. He is also grateful to Professor Ram Singh for his help and guidance.

\section{REFERENCES}

1. RAINVILLE, E.D., Special Functions, Chelsea Publishing Company, Bronx, New York, 1960 .

2. LEWIS, J.L., Convexity of Certain Series, J. London Math. Soc. 27(1983), 435-446.

3. LEWIS, J.L., Convolutions of Starlike Functions, Indiana Univ. Math. Jour. 27(4) $(1978), 671-688$.

4. STROHнÄCKER, E., Beitrage zur Theorie dee schlichten Funktionen, Math. Z. 37 $(1933), 356-380$. 


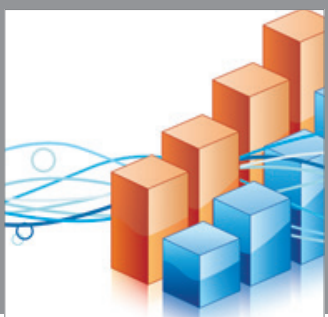

Advances in

Operations Research

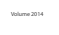

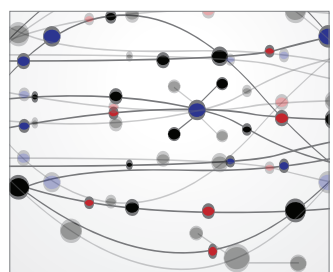

\section{The Scientific} World Journal
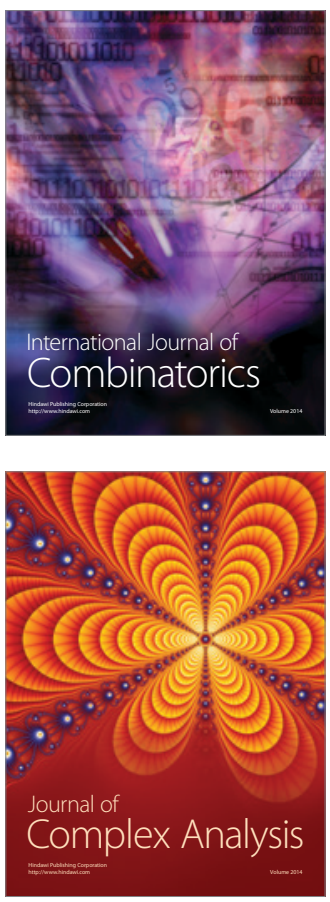

International Journal of

Mathematics and

Mathematical

Sciences
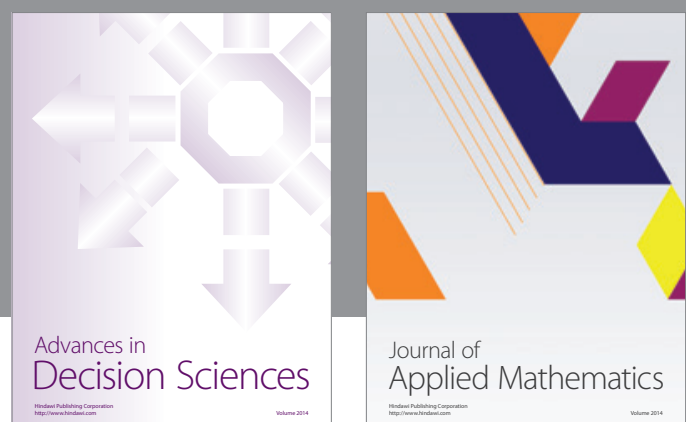

Journal of

Applied Mathematics
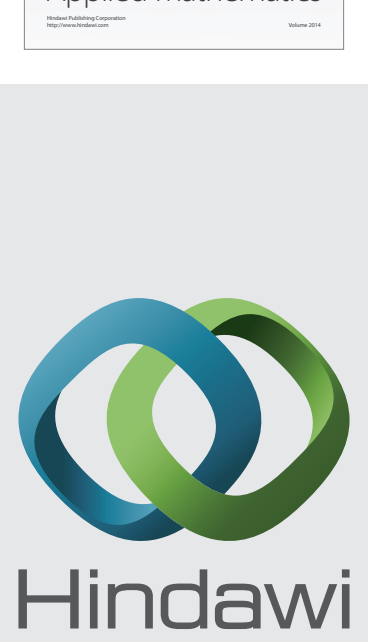

Submit your manuscripts at http://www.hindawi.com
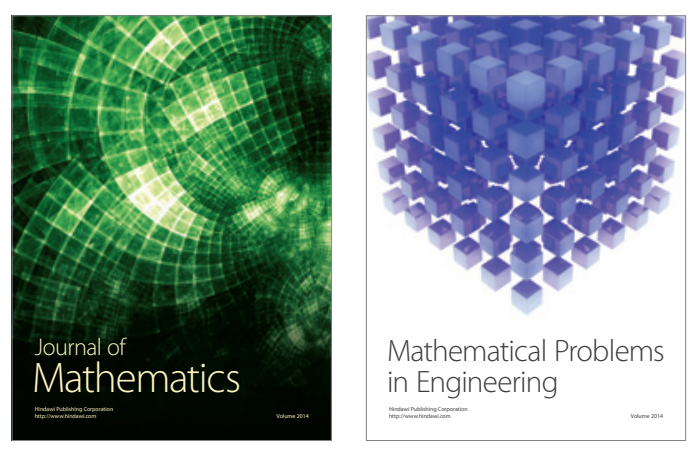

Mathematical Problems in Engineering
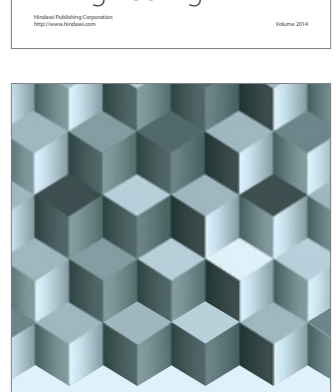

Journal of

Function Spaces
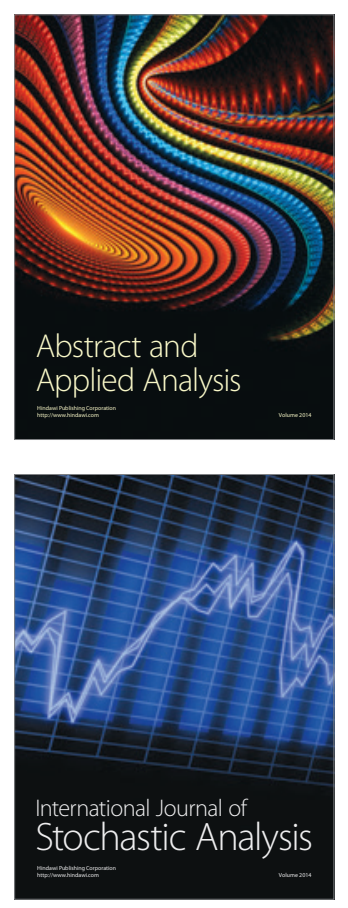

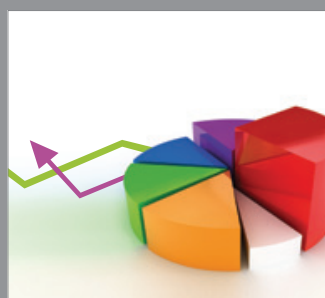

ournal of

Probability and Statistics

Promensencen
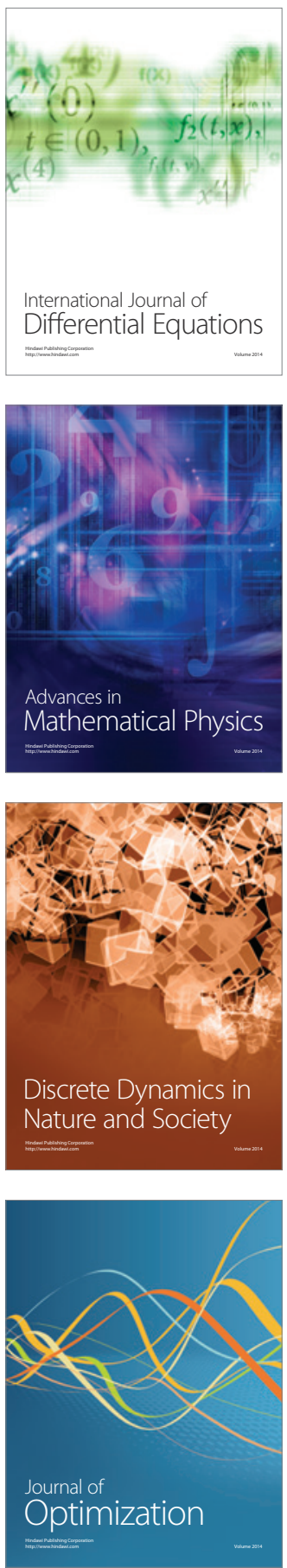